TITLE:

\title{
Numerical evaluation of vortex- induced vibration amplitude of a box girder bridge using forced oscillation method
}

\section{$\operatorname{AUTHOR}(S)$ :}

Noguchi, Kyohei; Ito, Yasuaki; Yagi, Tomomi

\section{CITATION:}

Noguchi, Kyohei ...[et al]. Numerical evaluation of vortex-induced vibration amplitude of a box girder bridge using forced oscillation method. Journal of Wind Engineering and Industrial Aerodynamics 2020, 196: 104029.

\section{ISSUE DATE:}

2020-01

URL:

http://hdl.handle.net/2433/260549

\section{RIGHT:}

(c) 2020. This manuscript version is made available under the CC-BY-NC-ND 4.0 license

http://creativecommons.org/licenses/by-nc-nd/4.0/; The full-text file will be made open to the public on 1 January 2022 in accordance with publisher's 'Terms and Conditions for Self-Archiving'.; この論文は出版社版でありません。引用の際 には出版社版をご確認ご利用ください。; This is not the published version. Please cite only the published version. 
1 Numerical evaluation of vortex-induced vibration amplitude of a box girder bridge using forced

4 Kyohei Noguchi

6

7

8

9

\section{oscillation method}

Yasuaki Ito

Tomomi Yagi

\section{Abstract}

\section{Keywords} number.

Kyoto University, Kyoto daigaku-katsura, Nishikyo-ku, Kyoto 615-8540, Japan

noguchi.kyohei.7z@kyoto-u.ac.jp

Corresponding author. Tel: +81 75383 3435; fax: +81753833168 .

Shimizu Corporation, 3-4-17, Etchujima, Koto-ku, Tokyo 135-8530, Japan

yasuaki.ito@shimz.co.jp

Kyoto University, Kyoto daigaku-katsura, Nishikyo-ku, Kyoto 615-8540, Japan

yagi.tomomi.7a@kyoto-u.ac.jp

The evaluation of the amplitude of the vortex-induced vibration (VIV) of a long-span bridge is necessary to implement a wind-resistant design. The development of high-performance computing has led to the use of computational fluid dynamics (CFD) in this domain, but the evaluation of VIV amplitude using the free vibration method in CFD incurs a high computational cost because of the small negative aerodynamic damping in the wind speed region of VIV. In this study, the use of flutter derivatives based on the forced oscillation method with a large eddy simulation is proposed for evaluating the VIV amplitude to reduce computational cost. The heaving VIV amplitude of a box girder was evaluated using simulated flutter derivatives and the results were validated by corresponding free vibration wind tunnel tests. Because the aerodynamic damping obtained by the flutter derivatives showed a clear dependence on the oscillation amplitude, the VIV amplitude can be evaluated using the proposed method. The effects of the spanwise domain size and Reynolds number were also significant.

Large Eddy Simulation, Flutter derivatives, Vortex-induced vibration, Spanwise domain size, Reynolds 


\section{Introduction}

Recent developments in high-performance computing have led to greater use of computational fluid dynamics (CFD) in engineering (Noguchi et al., 2017; Ishihara and Oka, 2018; Okaze et al., 2018; Zhou et al., 2018). In practice, however, CFD has not been adequately employed to achieve the wind-resistant design of a long-span bridge because aerodynamic stability as well as aerostatic stability must first be investigated. Some challenges persist in evaluating vortex-induced vibration (VIV) because calculating its amplitude and wind speed of occurrence is significantly important, which is different from the evaluation of flutter and galloping.

The VIV amplitude of a body is usually evaluated by the spring-supported free-vibration method in wind tunnel tests (Washizu et al., 1978; Miyata et al., 1983), and some researches have obtained this amplitude using the numerical free vibration method (Sun et al., 2008; Shimada and Ishihara, 2012; Nguyen et al., 2018; Álvarez et al., 2019). However, because the VIV amplitude increases (or decreases) gradually with time because of small aerodynamic damping, which makes it difficult to determine whether a steady-state response has been obtained, the free vibration method in CFD still incurs high computational costs. Thus, an alternative method is needed to evaluate the VIV amplitude using CFD for practical use.

Flutter derivatives have frequently been calculated based on the forced oscillation method (Sarpkaya, 1978; Staubli, 1983; Morse and Williamson, 2009) using CFD. This method can obtain aerodynamic forces in the periodic state in less time than the free vibration method, which reduces computational cost. For example, Šarkić et al. (2012) conducted unsteady Reynolds-Averaged Navier-Stokes (URANS) simulations for a box girder to obtain flutter derivatives. Using large eddy simulations (LES), Maruoka and Hirano (2000) focused on a thin plate, and Sarwar et al. (2008) and Ito and Graham $(2016,2017)$ conducted simulations of box girders to successfully calculate flutter derivatives with good accuracy. Because flutter derivatives can be used to evaluate aerodynamic damping, the VIV amplitude can also be evaluated using flutter derivatives by considering its dependence on the oscillation amplitude. However, because these authors focused on regions of relatively large wind speed to evaluate flutter instability, the possibility of evaluating the VIV amplitude using the forced oscillation method was not discussed. Sarwar and Ishihara (2010) evaluated the VIV using the forced oscillation method and obtained the wind speed region of VIV, but employed the free vibration method to evaluate the VIV amplitude. Thus, the forced oscillation method has not been often applied to calculate the VIV amplitude.

In light of the above, this study discusses the possibility of evaluating the VIV amplitude based on the forced oscillation method using LES. Because the inertia force does not act on the structure in simulations performed in CFD, unlike in wind tunnel tests, the forced oscillation method can calculate the aerodynamic forces accurately even in regions of low wind speed where the VIV appears. Moreover, because aerodynamic damping derived from the forced oscillation method does not depend on structural variables such as mass and damping, the simulated results can be used to calculate the VIV amplitude of a system with any Scruton number. The dependence of the aerodynamic damping term of the flutter 
derivatives on the amplitude of oscillation is first investigated in the wind speed region of VIV. Then, the VIV amplitude calculated by the aerodynamic damping based on the forced oscillation method using LES is compared with corresponding spring-supported free vibration wind tunnel tests to discuss its accuracy and validity. Further, the factors influencing aerodynamic damping and the VIV amplitude in the proposed method are investigated and the corresponding mechanisms are clarified.

\section{Experimental setup and numerical method}

Ito and Graham $(2016,2017)$ focused on the cross-section of a box girder bridge (Šarkić et al., 2012; Nieto at al., 2015) and accurately calculated the flutter derivatives using LES in a region of large reduced wind speed. In this study, the VIV amplitude was calculated using the same section on which the protuberances were additionally installed to excite aerodynamic vibrations for both the wind tunnel tests and LES (Nagao et al., 1997; Guan et al., 2012). Fig. 1 shows the cross-section of the target bridge in this study, the VIV amplitude of which with vertical one-degree-of-freedom (1DOF) was evaluated by wind tunnel tests and LES. Protuberances were installed upstream and downstream on the upper surface. The side ratio of the bridge section was $B / D=5.5$, where $B=$ deck width and $D=$ deck height excluding protuberances.

In this section, the experimental setup and the employed numerical method are first explained. Then, the definitions of the aerostatic and aerodynamic forces are provided. The aerostatic forces were calculated by both wind tunnel tests and numerical simulations to validate the numerical method employed in this study.

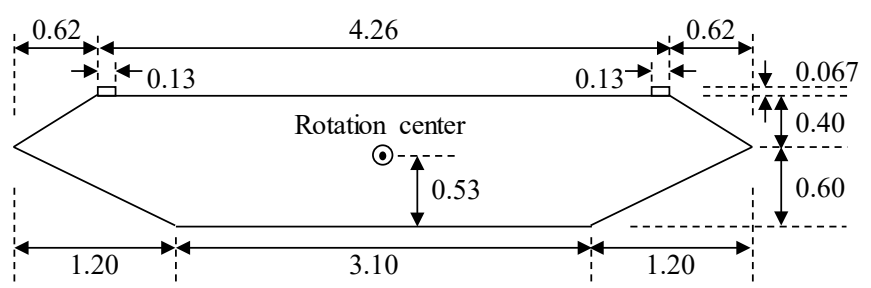

Fig. 1. Bridge section used for wind tunnel tests and LES (non-dimensionalized by deck height, $D$ ).

\subsection{Experimental setup}

The wind tunnel used was of the closed-circuit type located at Shimizu Corporation, and had a working section of $1.1 \mathrm{~m}$ in width and $0.9 \mathrm{~m}$ in height. The height $(D)$, width $(B)$, and length $(L)$ of the section model used in the experiment were $30 \mathrm{~mm}, 165 \mathrm{~mm}$, and 1,000 mm, respectively. All tests in this study were conducted under smooth flow conditions using this wind tunnel. The turbulence intensities of flow at $5 \mathrm{~m} / \mathrm{s}$ and $10 \mathrm{~m} / \mathrm{s}$ were $\sim 1 \%$, which can still be regarded as smooth in accordance with the HonshuShikoku Bridge Authority (2001). The spring-supported free vibration method was employed to obtain the VIV amplitude in the vertical 1DOF. The model was suspended using eight coil springs in the 
107

108 109

110

(a)

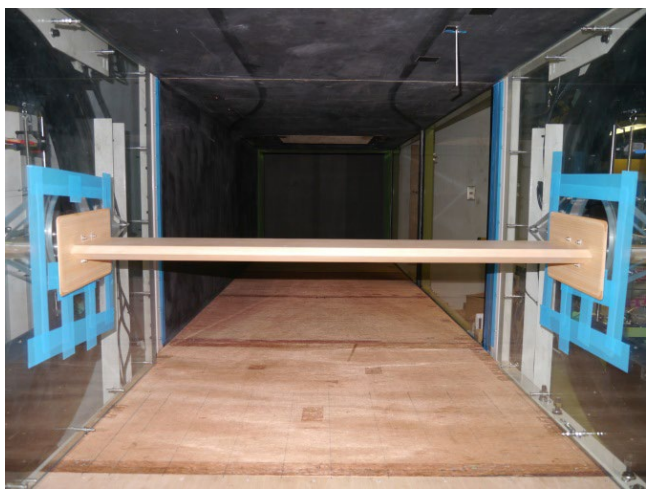

(b)

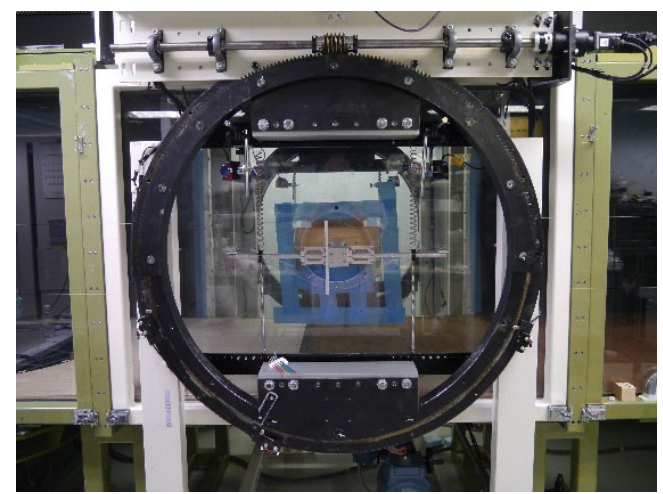

working section of the wind tunnel. The movements along the torsional and swaying directions were fixed to realize the vertical 1DOF condition. The experimental setup is shown in Fig. 2.

Fig. 2. Experimental setup. (a) front view, (b) side view.

The heaving natural frequency and equivalent mass were $3.104 \mathrm{~Hz}$ and $2.016 \mathrm{~kg}$, respectively. Fig. 3 shows the relationship between the logarithmic decrement in structural damping $(\delta)$ and nondimensional amplitude $(\eta / D)$, where $\eta=$ half amplitude of response, obtained from vibrations in calm conditions. Four similar trials were conducted, and the results of each are shown in Fig. 3. The logarithmic decrement was $\delta=0.0065$ (Scruton number $\left.S_{c}=2 m \delta /\left(\rho B^{2}\right)=0.80\right)$ at $\eta=3.0 \mathrm{~mm}(\eta / D=$ $0.10)$, where $m=$ mass of the system.

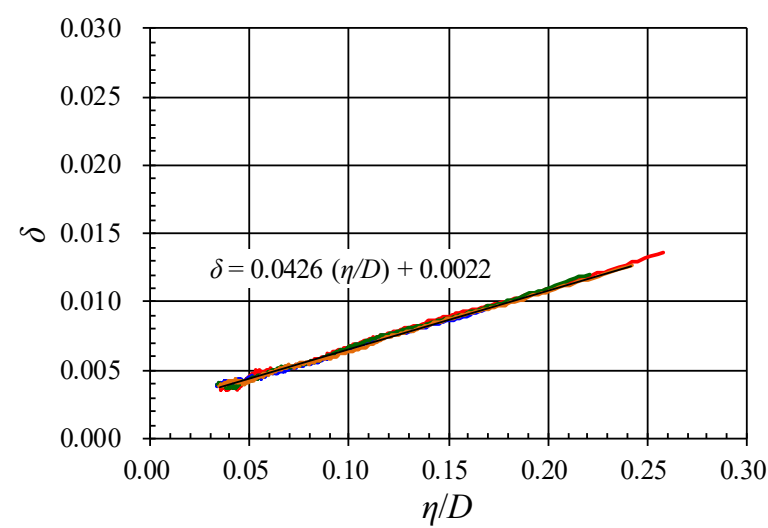

Fig. 3. Relationship between logarithmic decrement $(\delta)$ and amplitude $(\eta / D)$.

Moreover, the aerostatic force coefficients (mean wind force coefficients) were measured to validate the numerical simulation. Two three-component force balance devices (LMC-3501A-50N, Nissho Electric Works, Japan) were installed at the ends of the section model. The drag, lift, and pitching moment coefficients were calculated for every $2^{\circ}$ of the angle of attack from $-10^{\circ}$ to $10^{\circ}$. 
132 The numerical method used to calculate the flutter derivatives also followed Ito and Graham (2016,

133 2017), where the incompressible Navier-Stokes equation in generalized coordinates under the moving 134 grid condition was solved using the equation of continuity (Kajishima, 2014). A summary of the 135 numerical method is provided below. The fractional method was used for time advancement, the second136 order Adams-Bashforth scheme for the convection term, and the Crank-Nicolson scheme for the 137 diffusion term. The improved Morinishi method (Morinishi, 1995) was used for the discretization of the 138 convection term and the second-order central difference scheme for the other terms. A small amount of 139 numerical viscosity was added to the convection term to stabilize the simulation. The sub-grid scale 140 model was the standard Smagorinsky model $\left(C_{s}=0.12\right)$. The van Driest damping function was applied 141 around the wall surfaces.

142 Fig. 4 shows the computational domain. The structured O-type grid was used. The diameter of the 143 domain was $63.0 \mathrm{D}$ and the spanwise domain size was $1.0 \mathrm{D}$. The vertical size of the wall-adjacent grids 144 was $D / 400$ and the spanwise grid size was $D / 20$. The spanwise size of the grids used in this study was 145 sufficiently small according to Tamura et al. (1998). The number of grids in the circumferential, radial, 146 and spanwise directions were, respectively, 519, 264, and 21. 
148

(a)

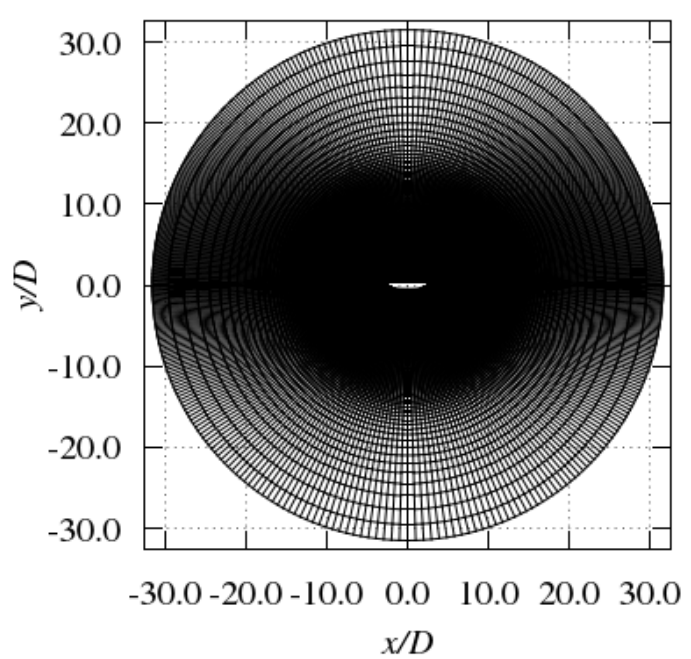

(b)

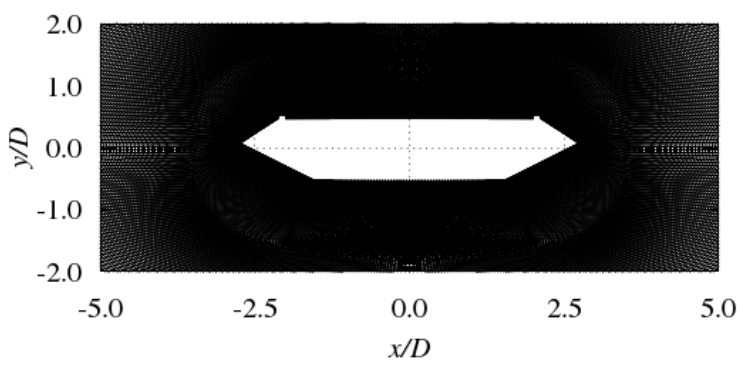

149

150

(c)

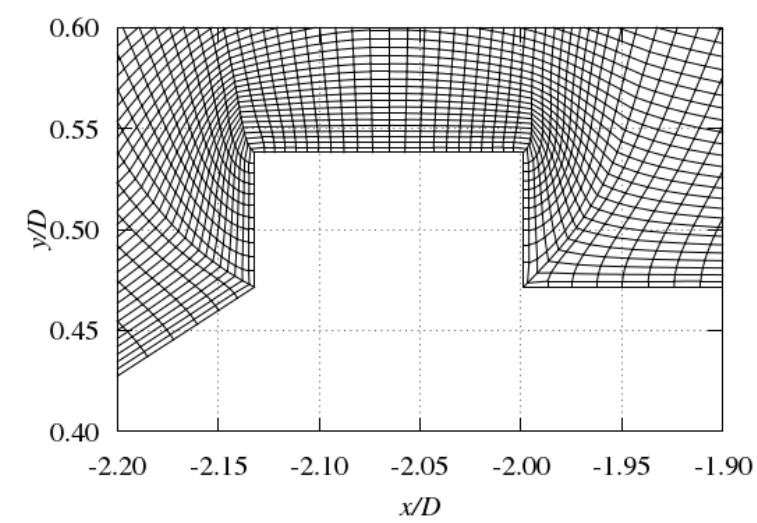

Fig. 4. Computational domain. (a) overall view, (b) enlarged view around the bridge section, (c) enlarged view around the protuberance.

The Reynolds number $(\operatorname{Re}=U D / v)$ was 20,000 , and the time increment was $3.0 \times 10^{-4}-8.0 \times 10^{-4}$ depending on the reduced wind speed $(U / f D)$ and the nondimensional oscillation amplitude $\left(\eta_{0} / D\right)$, where $U=$ wind speed, $v=$ kinematic viscosity, and $f=$ oscillation frequency. All the simulations were conducted under smooth flow conditions as with the wind tunnel tests. The flutter derivatives were calculated using 15 -cycle oscillations after a preliminary calculation of 5-15 cycle oscillations. U/fD was set to $6.0,8.0,9.0,10.0,11.0,12.0$, and 14.0 , and $\eta_{0} / D$ was set between 0.025 and 0.300 at intervals of 0.025 . Therefore, 84 cases with different combinations of $U / f D$ and $\eta_{0} / D$ were obtained. The case of $U / f D=10.0$ and $\eta_{0} / D=0.075$ yielded a nondimensional wall distance $\left(y^{+}\right)$between 0.9 and 1.5. This means that the wall-adjacent grids were within the laminar sub-layer and no special wall treatment was necessary. The maximum Courant number was 0.46 , and guaranteed a stable simulation (Ferziger and Perić, 2002). Moreover, the aerostatic force coefficients were calculated for every $2^{\circ}$ angle of attack from $-10^{\circ}$ to $10^{\circ}$, using 800 non-dimensional time after a preliminary calculation of 100 nondimensional time, for comparison with the results of the wind tunnel test for validation. 


\subsection{Definition of aerostatic force coefficients and flutter derivatives}

The time-averaged drag force $\left(D_{f}\right)$, lift force $\left(L_{f}\right)$, and pitching moment $\left(M_{f}\right)$ were defined using the aerostatic force coefficients (drag force $\left(C_{D}\right)$, lift force $\left(C_{L}\right)$, and pitching moment $\left(C_{M}\right)$ ) as below:

$$
D_{f}=1 / 2 \rho U^{2} C_{D} B L, \quad L_{f}=1 / 2 \rho U^{2} C_{L} B L, \quad M_{f}=1 / 2 \rho U^{2} C_{M} B^{2} L
$$

where $\rho=$ air density, $B=$ deck width, and $L=$ deck length. The directions of the wind forces were set as downstream-side positive for the drag force, upside positive for the lift force, and upstream-side up positive for the pitching moment.

The unsteady lift force $\left(L_{a e}\right)$ (downside positive) in the vertical 1 DOF was defined using two flutter derivatives as below (Scanlan and Tomko, 1971);

$$
L_{a e}=1 / 2 \rho U^{2} B L\left[K H_{1}^{*} \dot{\eta} / U+K^{2} H_{4}^{*} \eta / B\right]
$$

where $K=$ reduced frequency $(B \omega / U), \omega=$ circular frequency, and $\eta=$ heaving displacement (downside positive). $H_{1}^{*}$ and $H_{4}^{*}$ are the flutter derivatives used in this study to define the unsteady lift force. $H_{1}^{*}$ describes aerodynamic damping because it is included in the heaving velocity term, which is defined as below:

$$
H_{1}^{*}=-\frac{L_{\eta} \sin \Psi_{L}}{B /_{D} \times \widetilde{\omega}^{2} \times \eta_{0} /_{D}}
$$

where $L_{\eta}=$ nondimensional amplitude of the unsteady lift force, $\Psi_{L}=$ phase lag between heaving displacement (at the downward maximum) and unsteady lift force (at the downward maximum), and $\widetilde{\omega}$ $=$ nondimensional circular frequency $(=\omega D / U)$.

\section{Experimental and numerical results}

In this section, first, the numerical results of aerostatic force coefficients are compared with the experimental results to validate the numerical method. Second, the results of spring-supported wind tunnel tests are described and the characteristics of the observed aerodynamic vibrations are noted. Third, the aerodynamic damping obtained from the forced oscillation method is described with various combinations of reduced wind speed and oscillation amplitude. Finally, the dependence of the aerodynamic damping on the oscillation amplitude is explained to discuss the possibility of calculating the VIV amplitude.

\subsection{Aerostatic force coefficients}

Fig. 5 shows the aerostatic force coefficients obtained by the wind tunnel tests and numerical simulations. The simulated values were in good agreement with the experimental results. Therefore, the numerical method used in this study is sufficiently accurate to describe the basic characteristics of the wind 


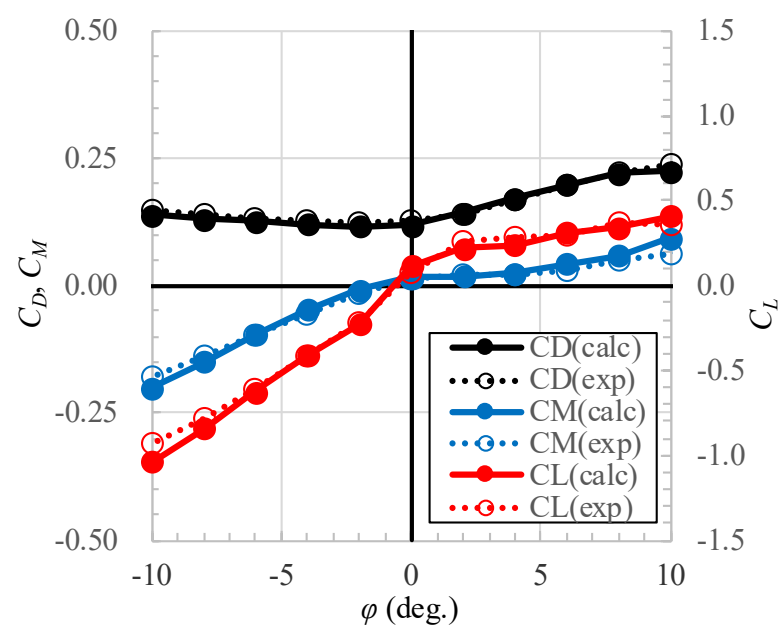

Fig. 5. Comparison of the aerostatic force coefficients obtained by the wind tunnel tests and numerical simulations.

\subsection{VIV obtained by the spring-supported free vibration wind tunnel tests}

Fig. 6 shows the experimental results of the spring-supported free vibration tests in the wind tunnel. VIVs were observed at $U / f D=5.0$ and 8.0-12.5; the maximum amplitude was $\eta / D=0.072$ at $U / f D=$ 10.9. According to Shiraishi and Matsumoto (1983), the critical reduced wind speed of the motioninduced-type VIV in the heaving mode was $U / f D=1.67 \times(1 / N) \times B / D$, where $N=$ natural number.

212 Because this equation yields $U / f D=4.6(N=2)$ and $9.2(N=1)$ for the bridge section studied, and were in good agreement with the results of the wind tunnel test, the aerodynamic vibrations shown in Fig. 6 are assumed to be of the motion-induced-type VIV.

215 By numerical simulations using the forced oscillation method with LES, the authors reproduced the VIV at $U / f D=8.0-12.5$ that yielded the maximum amplitude.

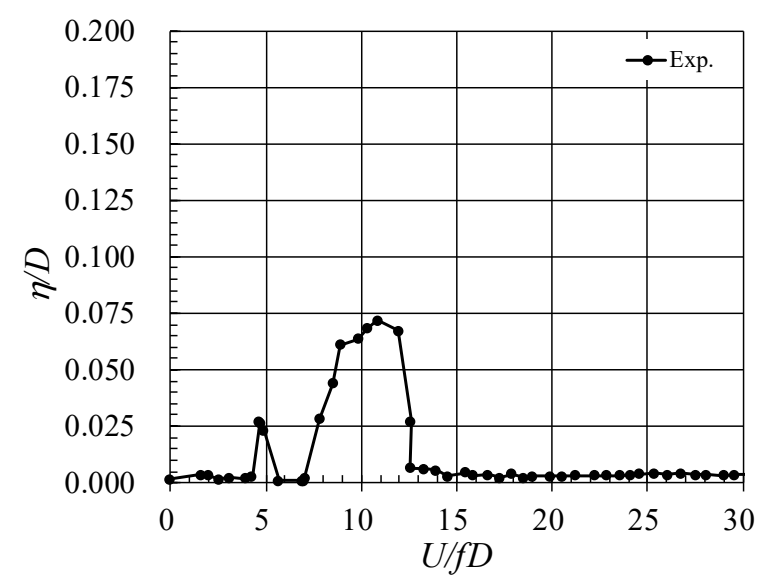

Fig. 6. Velocity-amplitude diagram obtained by the wind tunnel tests. 
Fig. 7 shows the flutter derivatives of heaving aerodynamic damping $\left(H_{1}^{*}\right)$ simulated by LES using the

222 forced oscillation method. Ito and Graham $(2016,2017)$ have shown that flutter derivatives simulated

223 in the same way as in this study are in good agreement with those obtained by wind tunnel tests. Thus,

224 a discussion based on this should be sufficiently reliable. Fig. 8 shows the results as a contour figure of

$225 H_{1}^{*}$ based on Fig. 7 to investigate the relationship among reduced wind speed, nondimensional

226 oscillation amplitude, and heaving aerodynamic damping. A positive $H_{1}^{*}$ means negative aerodynamic

227 damping and a negative $H_{1}^{*}$ indicates positive aerodynamic damping. $H_{1}^{*}$ in Fig. 8 shows a clear

228 dependence on the oscillation amplitude, especially at approximately $U / f D=8-13$, which corresponded

229 with the wind speed region of VIV by wind tunnel tests. Moreover, $H_{1}^{*}$, in an area of Fig. 8, was positive,

230 which suggests the occurrence of VIV.

231

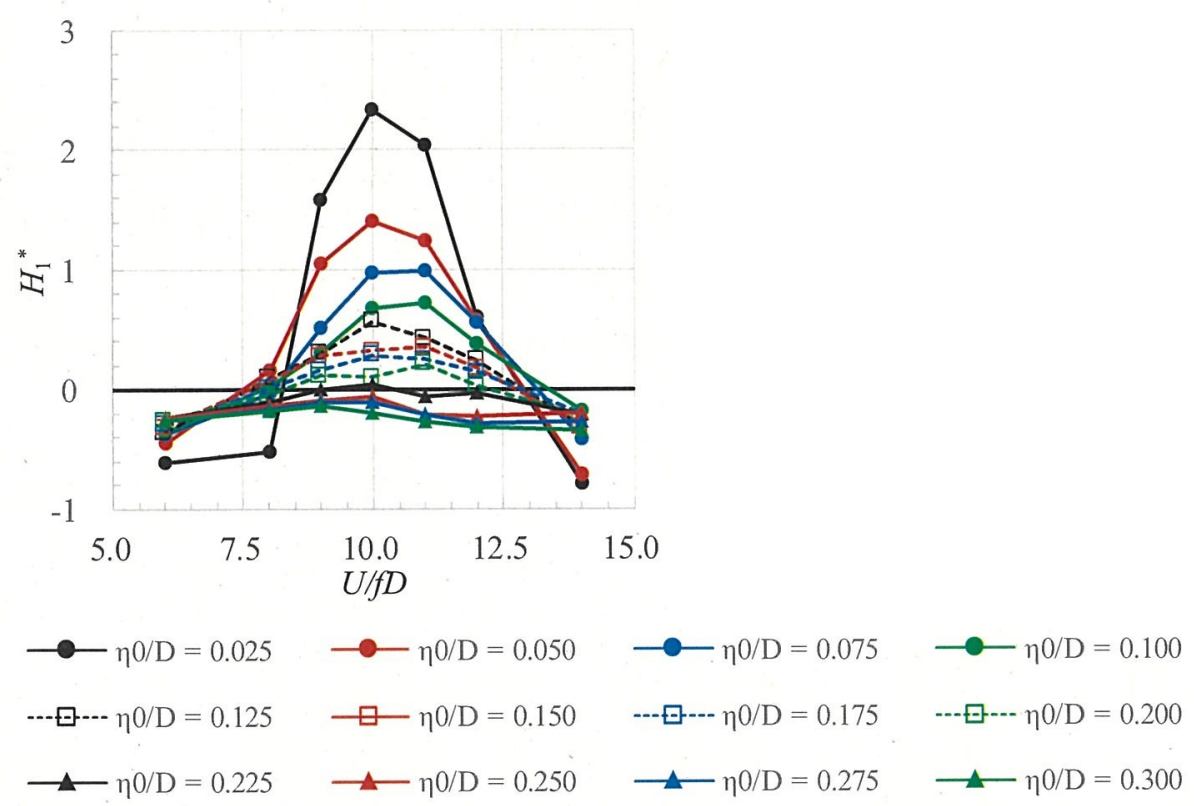

234 Fig. 7. Flutter derivatives of heaving aerodynamic damping $\left(H_{1}^{*}\right)$ for each nondimensional oscillation half amplitude $\left(\eta_{0} / D\right)$ as a function of reduced wind speed $(U / f D)$. 


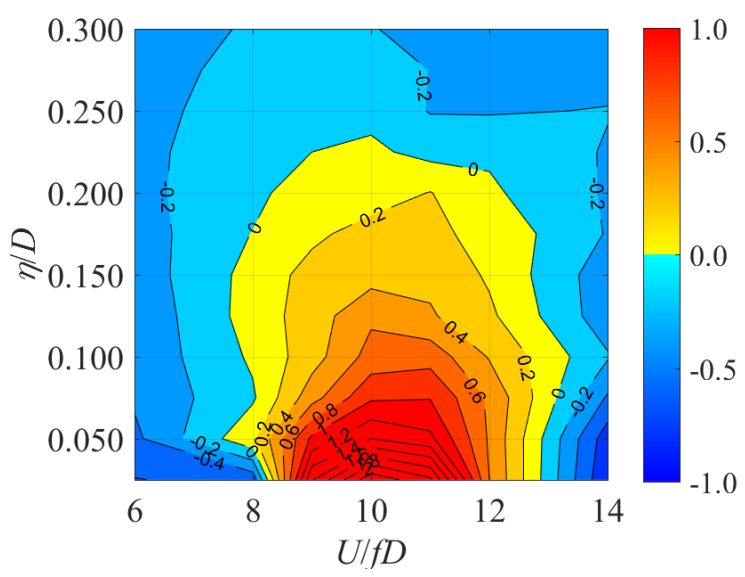

Fig. 8. Contour figure of aerodynamic damping $\left(H_{1}^{*}\right)$ as a function of reduced wind speed $(U / f D)$ and nondimensional oscillation half amplitude $\left(\eta_{0} / D\right)$.

Thus, the possibility of calculating the VIV amplitude using the proposed method has been shown, and incurs a lower computational cost than the free vibration method. In the next section, the VIV amplitude is calculated on the basis of aerodynamic damping as shown in Fig. 8 and compared with the results of the free vibration wind tunnel tests. Then, factors influencing aerodynamic damping are investigated to accurately evaluate the VIV amplitude.

\section{Discussion}

As mentioned in the previous section, aerodynamic damping based on the forced oscillation method using LES showed a clear dependence on the oscillation amplitude. In this section, first, the VIV amplitude is calculated using aerodynamic damping and compared with the results of free vibration wind tunnel tests to discuss the accuracy and validity of the proposed method. Following this, the effects of spanwise domain size and the Reynolds number on aerodynamic damping and the VIV amplitude are investigated, and their mechanisms are discussed in terms of unsteady pressure characteristics.

\subsection{VIV amplitude based on forced oscillation method using LES}

While aerodynamic damping was calculated as shown in Fig. 8, the damping of the bridge deck was determined on the basis of structural damping as well as the aerodynamic damping. Fig. 9 describes the damping of the system $\left(H_{1}^{*}-2 m \delta /\left(\pi \rho B^{2}\right)\right)$, where $m=$ mass of the system and $\delta=$ logarithmic decrement in structural damping. The logarithmic decrement is given by the relationship shown in Fig. 3. A positive $H_{1}^{*}-2 m \delta /\left(\pi \rho B^{2}\right)$ describes the excitation of the vibration and a negative sign the attenuation. $H_{1}^{*}-2 m \delta /\left(\pi \rho B^{2}\right)$ still exhibited a clear dependence on the oscillation amplitude, such as $H_{1}^{*}$, although structural damping rendered the area with positive values smaller than that in Fig. 8 . Further, because $H_{1}^{*}-2 m \delta /\left(\pi \rho B^{2}\right)$ was positive at approximately $U / f D=8-13$ and became negative at larger oscillation amplitude, the simulated results indicate the occurrence of the VIV. The simulated 
region of wind speed where the VIV appeared was in good agreement with that in the experiments. Thus, the forced oscillation method using CFD with LES can be used to calculate the wind speed region of VIV with adequate accuracy.

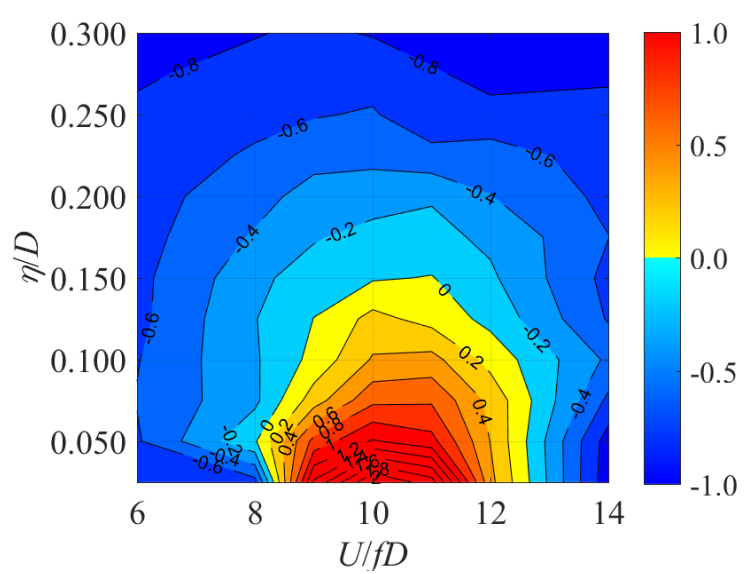

Fig. 9. Contour figure of damping of the system $\left(H_{1}^{*}-2 m \delta /\left(\pi \rho B^{2}\right)\right)$ as a function of reduced wind speed $(U / f D)$ and nondimensional oscillation half amplitude $\left(\eta_{0} / D\right)$.

Because the relationship among reduced wind speed, oscillation amplitude, and damping of the system was obtained as shown in Fig. 9, the amplitude of the steady-state response can be easily obtained by calculating the wind speed and amplitude that satisfy $H_{1}^{*}-2 m \delta /\left(\pi \rho B^{2}\right)=0$. The response amplitudes obtained are shown in Fig. 10 together with those from the free vibration wind tunnel tests. The VIV response was successfully evaluated, and the wind speed that yielded the maximum amplitude was comparable to that in the wind tunnel tests. Thus, the VIV amplitude can be determined by the forced oscillation method using LES as well as the wind speed region. This study is the first to adopt the forced oscillation method using CFD for calculating the VIV amplitude. Although the authors focus only on the motion-induced-type VIV of a box girder bridge, the proposed method can be widely employed to any kind of VIV because the method here follows those in previous research (Sarpkaya, 1978; Staubli, 1983; Morse and Williamson, 2009) that have employed experimental forced oscillation. Moreover, the damping of the system $\left(H_{1}^{*}-2 m \delta /\left(\pi \rho B^{2}\right)\right)$ can be written as $H_{1}^{*}-S_{c} / \pi$, where $S_{c}=$ the Scruton number. This means that aerodynamic damping derived from the forced oscillation method $\left(H_{1}^{*}\right)$ can be used for calculating the VIV amplitude with any $S_{c}$. Thus, the calculation of the VIV amplitude using the forced oscillation method has an advantage compared with the free vibration method.

However, the VIV amplitude simulated by LES was overestimated more than that by the experimental free vibration method because of very large negative aerodynamic damping. Thus, for the appropriate wind-resistant design of a long-span bridge, the causes of the discrepancy between the results of the wind tunnel tests and the numerical simulations should be clarified.

In the following sections, first, the effects of the size of the nondimensional spanwise domain $\left(L_{z} / D\right)$ are investigated. The characteristics of a flow field are different along the span due to threedimensionality. Therefore, the dependence of aerodynamic damping on $L_{z} / D$ should be examined. 
Second, the effects of the Reynolds number $(\mathrm{Re})$ are interesting to investigate. In wind tunnel tests and CFD analyses, Re is usually set to a smaller value, such as several tens of thousands, than that of a real bridge because the separation points are fixed at the corners of the bridge and, thus, the difference in Re between a prototype and an experimental or numerical model is expected to not affect the flow field. However, the Re of a wind speed region of VIV in wind tunnel tests is still small. Thus, the effects of Re on the VIV amplitude should be investigated to determine the validity of the numerical simulation used in this study, and to discuss the suitability of the VIV amplitude obtained from wind tunnel tests for the wind-resistant design.

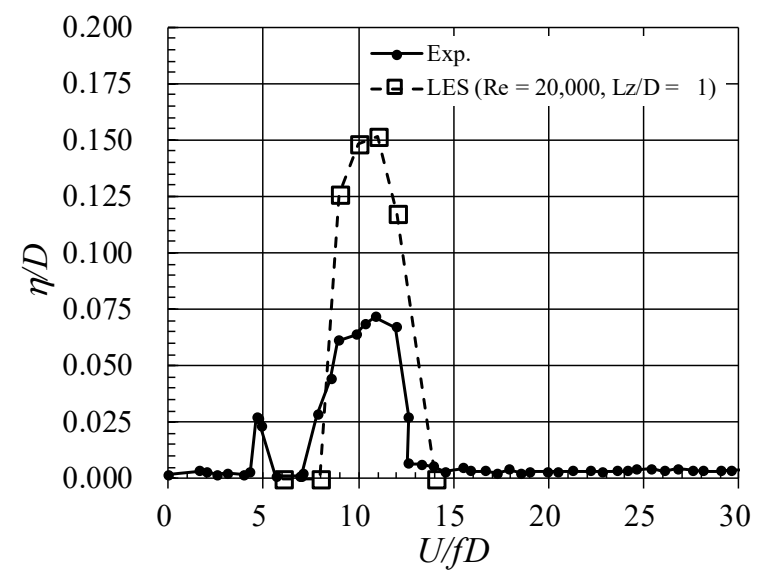

Fig. 10. Velocity-amplitude diagram obtained by the wind tunnel tests and LES.

\subsection{Effects of spanwise domain size}

\subsubsection{Characteristics of unsteady lift force}

The effects of the spanwise domain size $\left(L_{z} / D\right)$ on the flutter derivatives for heaving aerodynamic damping $\left(H_{1}^{*}\right)$ were investigated. The nondimensional spanwise domain size was set to $L_{z} / D=1.0,2.0$, $5.0,10.0$, and 20.0, respectively. The spanwise grid size was maintained as $D / 20$ for all cases; therefore, the numbers of grids in the spanwise direction were 21, 41, 101, 201, and 401. The oscillation amplitude was set to $\eta_{0} / D=0.075,0.100,0.125$, and 0.150 , and the reduced wind speed was fixed at $U / f D=10.0$. The other conditions were the same as in the previous section.

As shown in Fig. 11(a), $H_{1}^{*}$ tended to decrease with increasing $L_{z} / D . H_{1}^{*}$ also exhibited an asymptotic trend against $L_{z} / D$. Ito and $\operatorname{Graham}(2016,2017)$ observed a similar trend for aerostatic force coefficients at $L_{z} / D=10-20$ because of the 3-D flow field. The same phenomenon was observed in this study as well. Thus, $L_{z} / D$ affects aerodynamic damping and, consequently, the VIV amplitude. The amplitude for $U / f D=10.0$ was $\eta / D=0.133$ in case of $L_{z} / D=20.0$ while $\eta / D=0.148$ in case of $L_{z} / D=$ 1.0. To explore the mechanisms, the effects of $L_{z} / D$ on the amplitude $\left(L_{\eta}\right)$ and phase lag $\left(\Psi_{L}\right)$ of the unsteady lift force, which directly influenced $H_{1}^{*}$ (Eq. (3)), were also investigated as shown in Figs. 11(b) and 11(c). The amplitude of the unsteady lift force $\left(L_{\eta}\right)$ tended to decrease with increasing $L_{z} / D$, 
326

327

328

329

330

331

332

(a)

334 (c)
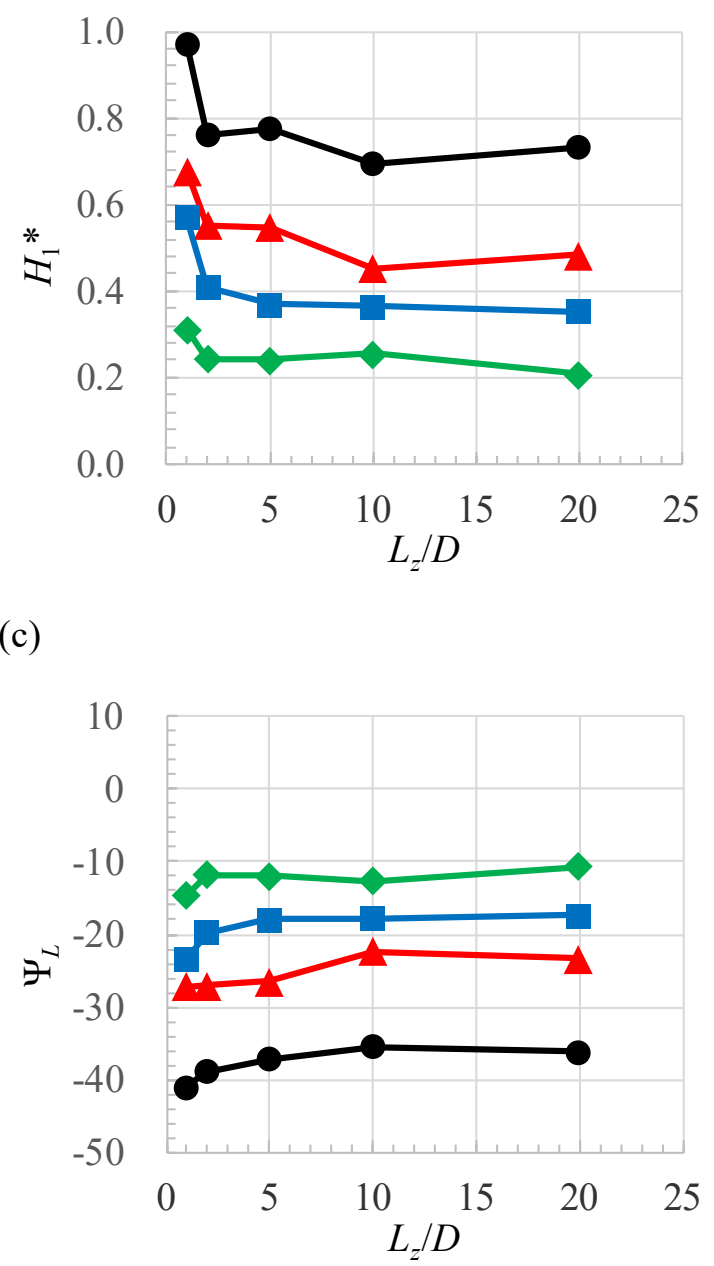

\subsubsection{Characteristics of unsteady pressure}

especially in the range $L_{z} / D=1.0-2.0$. The phase lag $\left(\Psi_{L}\right)$ shows negative values for all cases, which indicates the excitation of the vibration. The absolute value of $\Psi_{L}$ exhibited a clear tendency to decrease with increasing $L_{z} / D$. Because a small amplitude and phase lag of the unsteady lift force reduce the aerodynamic damping as well, $H_{1}^{*}$ also decreased with an increase in $L_{z} / D$. Thus, a sufficiently large spanwise domain size is necessary to accurately evaluate the VIV amplitude.

(b)

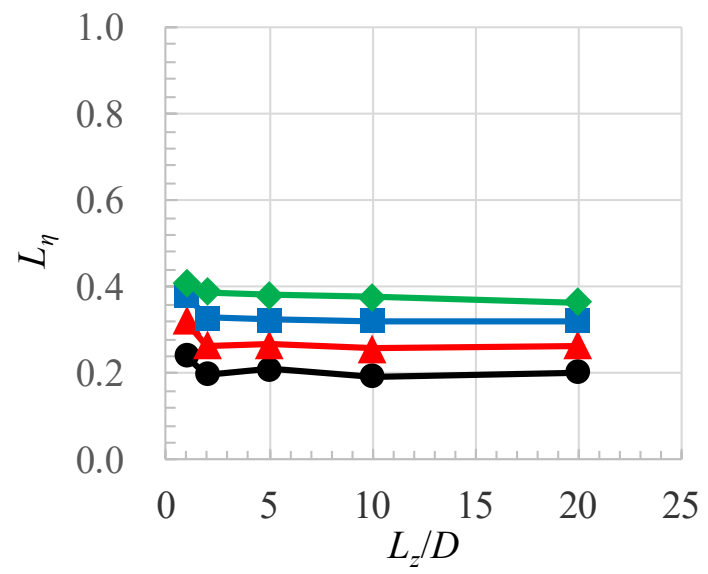

$\rightarrow \eta 0 / \mathrm{D}=0.075 \multimap \eta 0 / \mathrm{D}=0.100 \rightarrow \eta 0 / \mathrm{D}=0.125 \rightarrow \eta 0 / \mathrm{D}=0.150$

Fig. 11. Effects of spanwise domain size on unsteady lift force. (a) aerodynamic damping $\left(H_{1}^{*}\right)$, (b) amplitude $\left(L_{\eta}\right)$ of unsteady lift force, $(\mathrm{c})$ phase lag $\left(\Psi_{L}\right)$ of unsteady lift force.

The mechanisms of the effect of spanwise domain size $\left(L_{z} / D\right)$ on the characteristics of unsteady lift force were examined in detail. Because the unsteady lift force was calculated by integrating pressure around the bridge section, discussing the characteristics of unsteady pressure is important and suggestive.

The forced oscillation method was employed under $L_{z} / D=1.0$ or $10.0, \eta_{0} / D=0.075$, and $U / f D=$ 10.0. $L_{z} / D=10.0$ was employed because $H_{1}^{*}$ converged to a certain value at $L_{z} / D=10-20$ as mentioned 
above. Spanwise-averaged unsteady wall pressures at every five grids along the circumferential direction were obtained. Then, the nondimensional half amplitude of unsteady pressure $\left(\tilde{C}_{p}\right)$ and phase lag $\left(\Psi_{p}\right)$ at each grid were evaluated. The phase lag was defined as a difference in phase between the heaving displacement (at the downward maximum) and the unsteady pressure (with the maximum positive value on the upper surface and the maximum negative value on the lower surface, both of which resulted in a downward lift force). Finally, aerodynamic damping in the heaving mode at each grid $\left(\tilde{C}_{p H_{1}^{*}}\right)$, and the relationship between $\tilde{C}_{p H_{1}^{*}}$ and $H_{1}^{*}$ can be written as below (Matsumoto et al., 1996).

$$
H_{1}^{*}=\frac{1}{B / D \times \widetilde{\omega}^{2} \times \eta_{0} / D} \int-\tilde{C}_{p} \sin \Psi_{p} d x=\frac{1}{B / D \times \widetilde{\omega}^{2} \times \eta_{0} / D} \int \tilde{C}_{p H_{1}^{*}} d x
$$

These definitions of the parameters prompt a similar discussion with the characteristics of unsteady lift force of $L_{\eta}, \Psi_{L}$, and $H_{1}^{*}$. A positive $\Psi_{p}$ results in a negative $\tilde{C}_{p H_{1}^{*}}$ (positive aerodynamic damping = attenuation of vibration), and a negative $\Psi_{p}$ leads to a positive $\tilde{C}_{p H_{1}^{*}}$ (negative aerodynamic damping = excitation of vibration). Considering these parameters at each grid on the girder, the difference in the pressure characteristics and, thus, the flow field caused by $L_{z} / D$ can be discussed in detail.

Fig. 12 shows the characteristics of unsteady pressure obtained, the lateral axis of which is the nondimensional horizontal coordinate $(x / D)$. The values of both the upper surface $\left(\boldsymbol{\Lambda}: L_{z} / D=10.0, \triangle\right.$ : $\left.L_{z} / D=1.0\right)$ and the lower surface $\left(\boldsymbol{\nabla}: L_{z} / D=10.0, \nabla: L_{z} / D=1.0\right)$ are plotted in each figure. The amplitude of pressure $\left(\tilde{C}_{p}\right)$ in $L_{z} / D=1.0$ tended to be higher in both the upper and the lower surfaces. Whereas the phase lag $\left(\Psi_{p}\right)$ of the upper surface is almost identical for the two cases, that of the lower surface in $L_{z} / D=1.0$ has a slightly larger area with negative values around $x / D=0$. The characteristics of $\tilde{C}_{p}$ and $\Psi_{p}$ resulted in those of $\tilde{C}_{p H_{1}^{*}}$, showing larger values of $L_{z} / D=1.0$ in both the upper and lower surfaces, especially in the downstream side and, thus, exhibited greater instability. In other words, $L_{z} / D$ affected the flow field around the bridge deck, especially downstream. These characteristics of the unsteady pressure explain those of the unsteady lift force as shown in Fig. 11 well. 
369

(a)

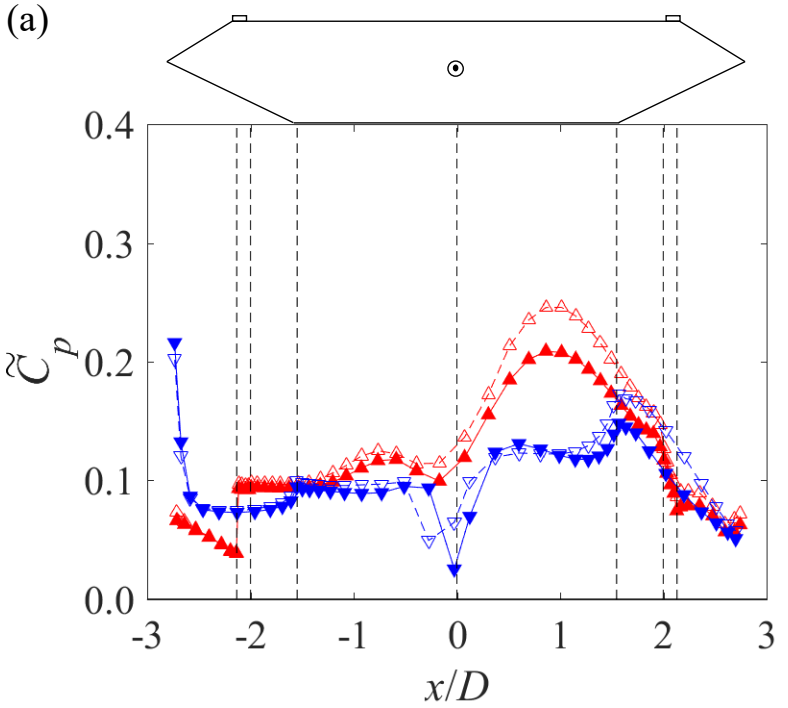

370

371 (c)

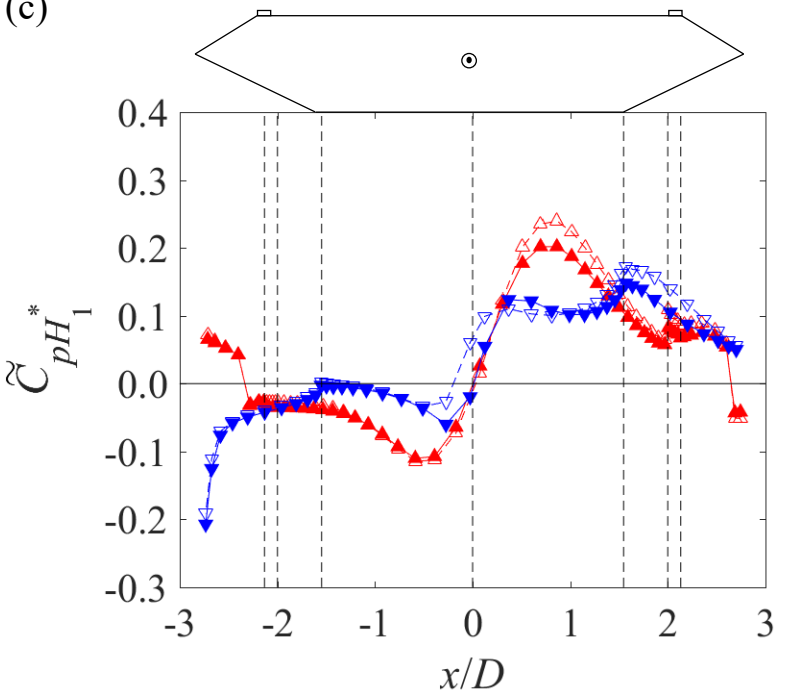

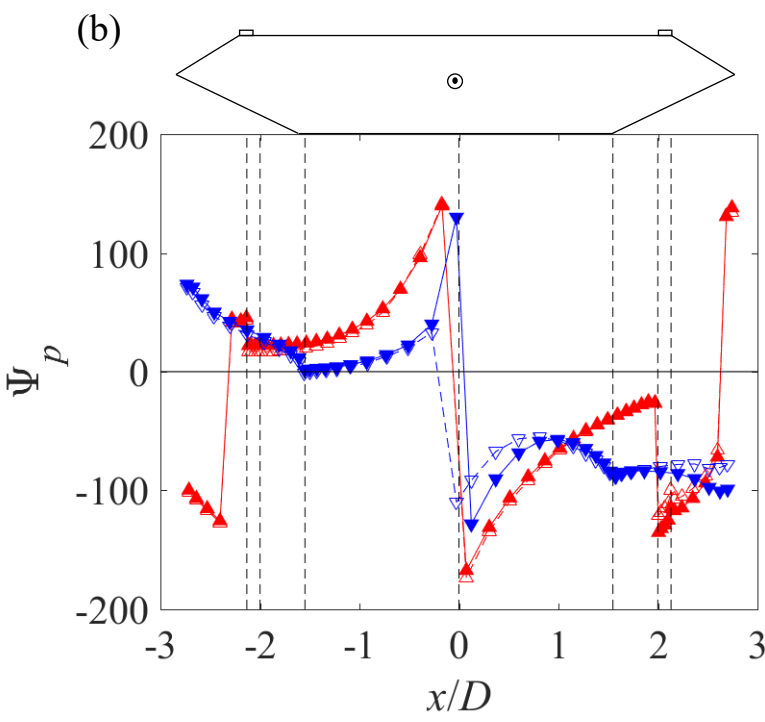

Fig. 12. Effects of spanwise domain size on unsteady pressure at each location. (a) amplitude $\left(\tilde{C}_{p}\right)$ of unsteady pressure, (b) phase lag $\left(\Psi_{p}\right)$ of unsteady pressure, (c) aerodynamic damping $\left(\tilde{C}_{p H_{1}^{*}}\right)$.

Moreover, the correlation coefficient of the oscillation frequency of pressure along the span at $x / D$ $=0$ was investigated in case of $L_{z} / D=1.0$ and 10.0. Pressure in this discussion is defined as the difference between pressure at the upper and lower surfaces. The correlation of the unsteady lift forces exhibited the same relations as that of the unsteady pressures. Fig. 13 shows the correlation coefficient of pressure at $z / D=0$ and $z / D=r / D$, where $r=$ distance from $z / D=0$. The correlation coefficient was large because of the oscillation. On the contrary, the correlation coefficient tended to become smaller with increasing $r / D$, which indicates a significant three-dimensionality of the flow field. This also indicates that a sufficiently large $L_{z} / D$ is necessary to consider the 3-D flow field. The overestimated correlation of flows in the spanwise direction in $L_{z} / D=1.0$ is considered to have affected the flow field around the bridge section, as shown in Fig. 11, which resulted in a larger VIV amplitude.

Thus, a sufficiently large size of the spanwise domain is required to consider the 3-D flow field for 
a proper evaluation of aerodynamic damping and the VIV amplitude. Although a larger spanwise domain increases computational cost, the free vibration method also requires this size to accurately evaluate the phenomena. This is because $L_{z} / D$ depends on the target flow field and not the methodology of simulation. Thus, the forced oscillation method still has an advantage in terms of computational cost against the free vibration method.

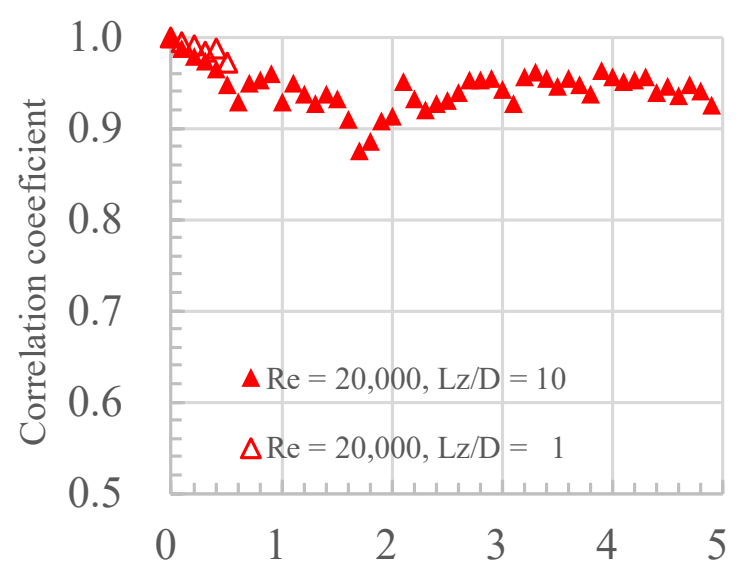

$r / D$

Fig. 13. The correlation coefficient of pressure along the span at $x / D=0$.

\subsection{Effects of Reynolds number}

The previous section clarified that a sufficiently large domain is necessary to implement the threedimensionality of the flow field. The section below discusses the effects of Re on the VIV amplitude in terms of the characteristics of unsteady lift force and unsteady pressure.

\subsubsection{Characteristics of unsteady lift force}

The effects of Re on $H_{1}^{*}$ were investigated at $L_{z} / D=10.0, \eta_{0} / D=0.075,0.100,0.125$, and 0.150 , and $U / f D=10.0$. Re was set to $\operatorname{Re}=20,000$ and 2,000. $\operatorname{Re}=20,000$ was the same value as in the former sections, and $\operatorname{Re}=2,000$ was selected because it corresponded with the occurrence wind speed of the VIV in the free vibration wind tunnel tests.

As shown in Fig. 14(a), Re affected $H_{1}^{*}$ and, consequently, the VIV amplitude; the amplitude for $U / f D=10.0$ and $L_{z} / D=10.0$ was $\eta / D=0.107$ in case of $\operatorname{Re}=2,000$, and $\eta / D=0.136$ in case of $\operatorname{Re}=$ 20,000. To explore the mechanisms, effects of Re on amplitude $\left(L_{\eta}\right)$ and phase lag $\left(\Psi_{L}\right)$ of the unsteady lift force were examined as shown in Figs. 14(b) and 14(c). Values of $L_{\eta}$ at $\operatorname{Re}=2,000$ were larger than those at $\operatorname{Re}=20,000$. On the contrary, although $\Psi_{L}$ showed negative values for all cases, the absolute values of $\Psi_{L}$ in $R e=2,000$ were clearly smaller. As indicated by Eq. (3), a large amplitude and a large absolute value of negative phase lag result in a large negative aerodynamic damping (i.e., positive $H_{1}^{*}$ ). Thus, although $L_{\eta}$ and $\Psi_{L}$ in $\operatorname{Re}=2,000$ had opposite roles, the aerodynamic damping of each oscillation amplitude in $\operatorname{Re}=2,000$ was smaller because the effect of $\Psi_{L}$ seemed to be dominant. Thus, considering the effect of Re was very important to reproduce the VIV amplitude obtained from wind tunnel tests. 
416

(a)

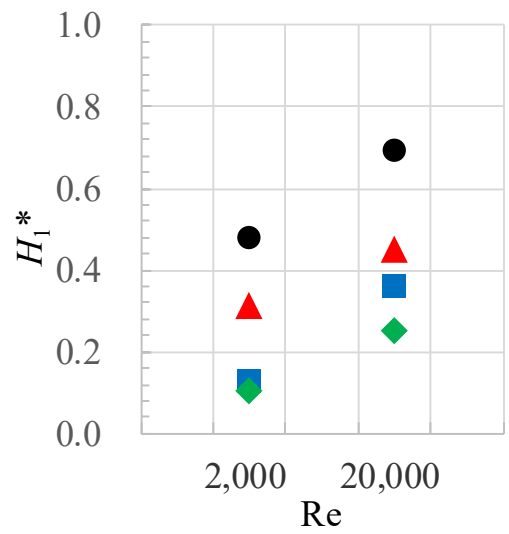

(b)

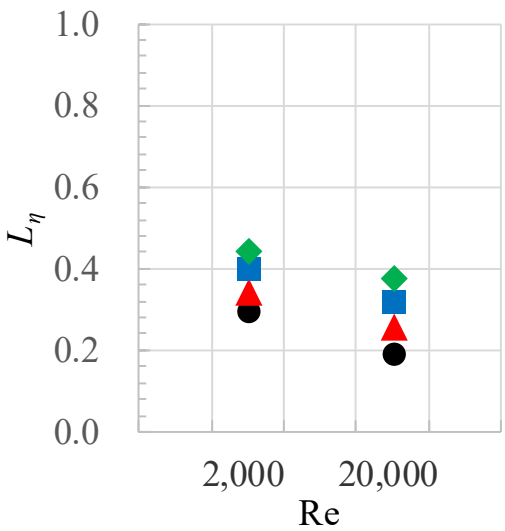

(c)

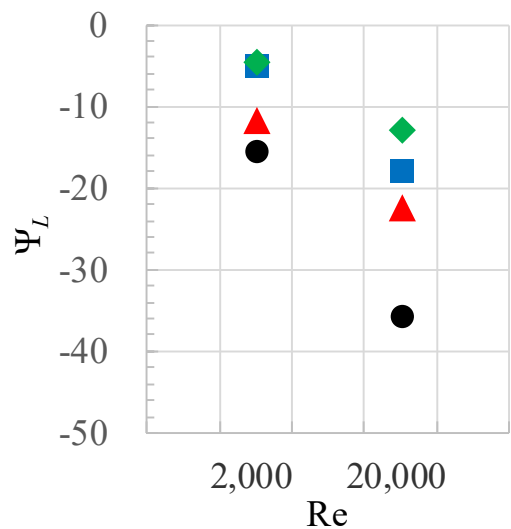

$\rightarrow 00 / \mathrm{D}=0.075$

$\simeq \eta 0 / \mathrm{D}=0.100$

$\rightarrow \eta 0 / \mathrm{D}=0.125 \rightarrow \eta 0 / \mathrm{D}=0.150$

Fig. 14. Effects of Reynolds number on unsteady lift force. (a) aerodynamic damping $\left(H_{1}^{*}\right)$, (b) amplitude

420 $\left(L_{\eta}\right)$ of unsteady lift force, (c) phase lag $\left(\Psi_{L}\right)$ of unsteady lift force,

421

\subsubsection{Characteristics of unsteady pressure}

423

The characteristics of unsteady pressure were also investigated to discuss the mechanisms of the effects of Re on the characteristics of unsteady lift force.

Fig. 15 shows the obtained characteristics of unsteady pressure. The values of both the upper surface $(\boldsymbol{\Delta}: \operatorname{Re}=20,000, \triangle: \operatorname{Re}=2,000)$ and the lower surface $\left(\boldsymbol{\nabla}: \operatorname{Re}=20,000, \nabla: L_{z} / D=\operatorname{Re}=2,000\right)$ are plotted. First, in the upper surface, $\tilde{C}_{p}$ for $\mathrm{Re}=20,000$ tended to be smaller, and $\Psi_{p}$ had a slightly larger area with negative values for $\operatorname{Re}=20,000$. Therefore, although the peak value of $\tilde{C}_{p H_{1}^{*}}$ for $\operatorname{Re}=2,000$ was larger, $\operatorname{Re}=20,000$ gave a larger area of $\tilde{C}_{p H_{1}^{*}}$ with positive values. Second, in the lower surface, $\tilde{C}_{p}$ for $\operatorname{Re}=20,000$ also tended to be smaller. On the contrary, $\Psi_{p}$ in the downstream side for $\operatorname{Re}=$ 43120,000 indicated significantly larger negative values than those for $\operatorname{Re}=2,000$. Therefore, $\tilde{C}_{p H_{1}^{*}}$ for $\operatorname{Re}$ $432=20,000$ had larger values over the downstream side, which appeared to result in larger $H_{1}^{*}$ in $\operatorname{Re}=$ 43320,000 . These characteristics of unsteady pressure explain well the difference in the characteristics of unsteady lift force between Reynolds numbers. 
436

(a)

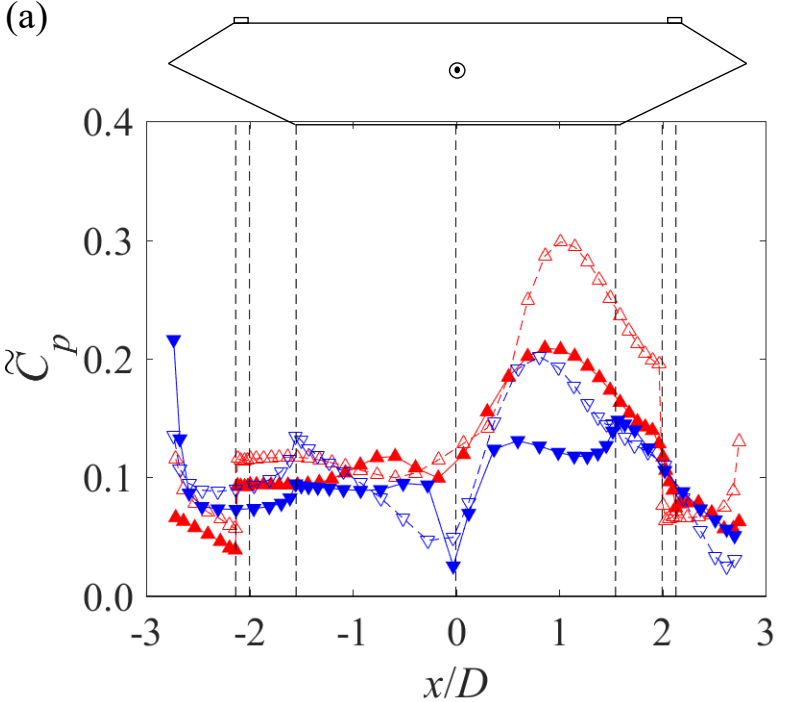

437

438

439

440

441

442

443

444

445

446

447

448

449

450

451

452

453

(c)

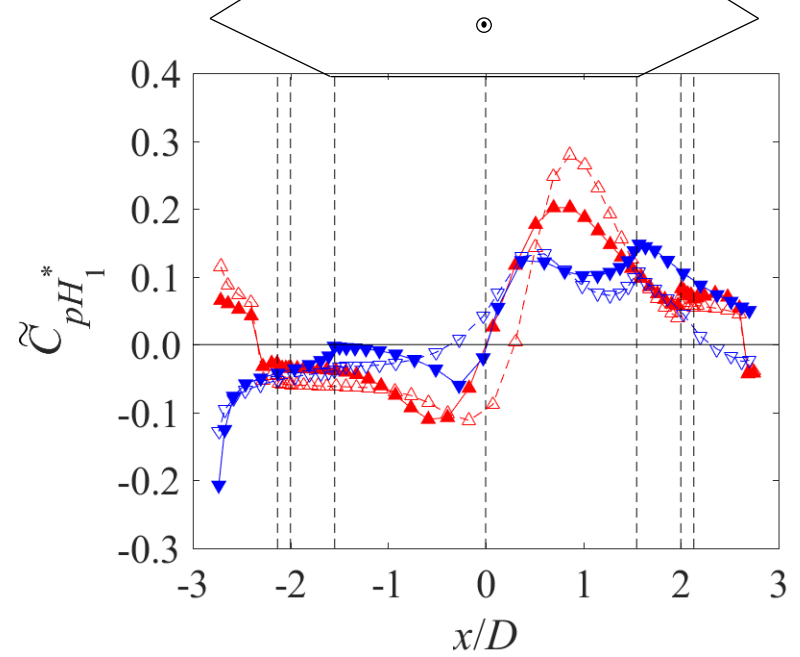

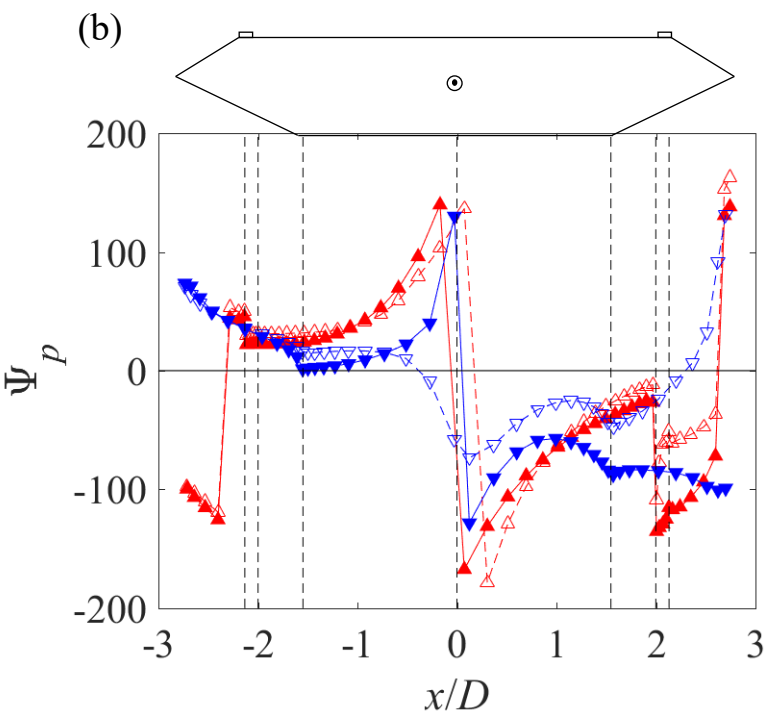

\footnotetext{
$\triangle \mathrm{Re}=20,000$ (upper) $\quad-\mathrm{Re}=20,000$ (lower)

$-\triangle-\operatorname{Re}=2,000$ (upper) $\quad-\nabla-\operatorname{Re}=2,000$ (lower)
}

Fig. 15. Effects of Reynolds number on unsteady pressure. (a) amplitude $\left(\tilde{C}_{p}\right)$ of unsteady pressure, (b) phase lag $\left(\Psi_{p}\right)$ of unsteady pressure, $(\mathrm{c})$ aerodynamic damping $\left(\tilde{C}_{p H_{1}^{*}}\right)$.

Fig. 16 shows the time-averaged pressure contours and velocity magnitude isograms for $\operatorname{Re}=20,000$ and $\operatorname{Re}=2,000$, respectively. The difference in the flow field between values of $\operatorname{Re}$ at the upper surface was small because the separation point was fixed on the protuberance and, thus, the flow field on the upper surface was insensitive to Re. On the contrary, the difference on the lower side was relatively large, including at the reattachment point because the lower side had a streamlined form, because of which a slight change in the separation point had a large influence on the flow filed. This is also obtained because flows at $\operatorname{Re}=20,000$ featured more turbulence than those at $\operatorname{Re}=2,000$, which caused earlier reattachment (Laneville et al., 1975). Thus, the numerical results reproduced the qualitative characteristics well, although discussing the discrepancy between the wind tunnel tests and LES is necessary. These characteristics of the flow field correspond with those of unsteady pressure shown in Fig. 15, in which the effects of Re are larger at the lower surface than at the upper surface. As a result, 
(a)

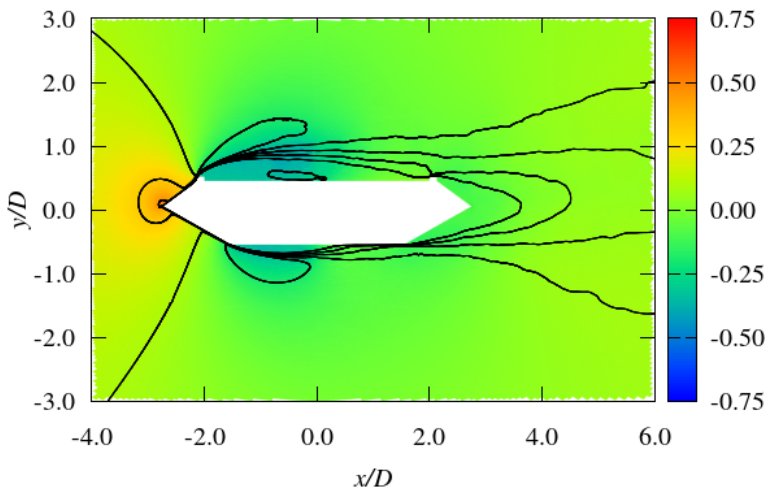

(b)

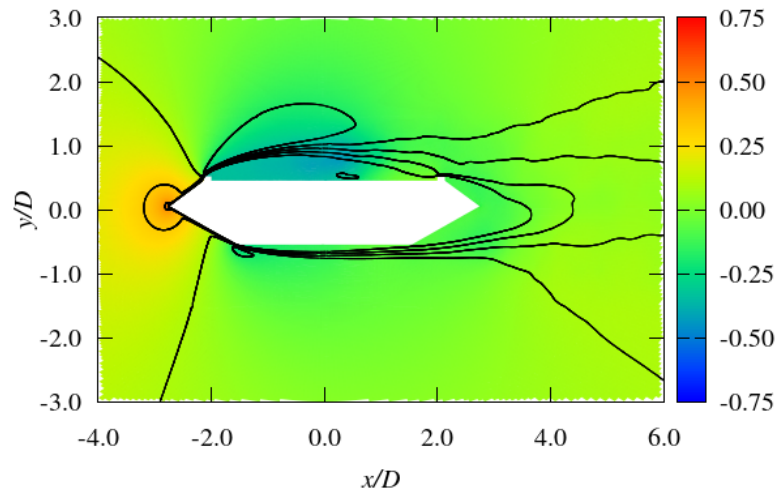

458

460

461

462

463

464

465

466

467

468

469

470

471

472

473

474

475

476

477

478

479

480

481

482

483

484

the effects of the Reynolds number on the flow field were significant, and thus the unsteady lift force and VIV amplitude were significantly different between $\mathrm{Re}=20,000$ and $\mathrm{Re}=2,000$.

Fig. 16. Time-averaged pressure contours and velocity magnitude isograms (colormap $=$ mean pressure, lines $=$ mean velocity magnitude isograms). (a) $R e=20,000$, (b) $R e=2,000$.

As discussed above, the flow fields around the bridge section at $\operatorname{Re}=20,000$ and 2,000 were different, especially downstream, and this resulted in a difference in the unsteady lift force, aerodynamic damping, and the VIV amplitude. Thus, the Reynolds number should be attended to, especially the reproduction of the VIV amplitude obtained from wind tunnel tests in case of a small Reynolds number. In other words, the considered case in this study implies as a possibility that the VIV amplitude calculated by wind tunnel tests may be affected by the Reynolds number and, thus, underestimated compared with that obtained from the prototype. This is because a small Reynolds number sometimes has to be employed for the wind-resistant design because of the limitation of experimental facilities. Thus, the experimental results of the VIV should be carefully interpreted. However, because the dependence of the VIV amplitude on the Reynolds number can be affected by the shape of the bridge section, including the existence of such attachments as handrails, further investigations are needed for a comprehensive understanding of the numerical performance and accuracy of the wind tunnel tests, especially at low Reynolds numbers.

\subsection{Comparison of VIV amplitude obtained by wind tunnel tests and LES}

Finally, the VIV amplitude obtained by wind tunnel tests and numerical simulations using LES were compared as shown in Fig. 17. The reduced wind speed was set to $U / f D=10.0$. As mentioned above, the size of the spanwise domain and Re should be appropriately determined to better calculate the VIV amplitude. In particular, the effect of Re is more significant than that of the size of the spanwise domain. However, even if the effects of Re and domain size are considered, the VIV amplitude obtained by the forced oscillation method using LES is still larger than that obtained from the free vibration wind tunnel tests. 
494

495

The spanwise size of the grids used in this study was determined according to Tamura et al. (1998). However, as mentioned by Bruno et al. (2012), the spanwise discretization may play a great role in the accuracy of the simulations. Therefore, the effects of the spanwise discretization should be investigated in future work. Moreover, the small turbulence in the approaching flow of the wind tunnel tests might have reduced amplitude. For example, Wardlaw et al. (1983) noted that the VIV amplitude of a bride deck decreased dramatically under turbulent flow. In addition, the accuracy of the wind tunnel tests was not necessarily adequate because the measurements were carried out in regions of low wind speed. Additional numerical simulations using LES are thus necessary in future work to further investigate the effects of turbulent flow on the VIV amplitude.

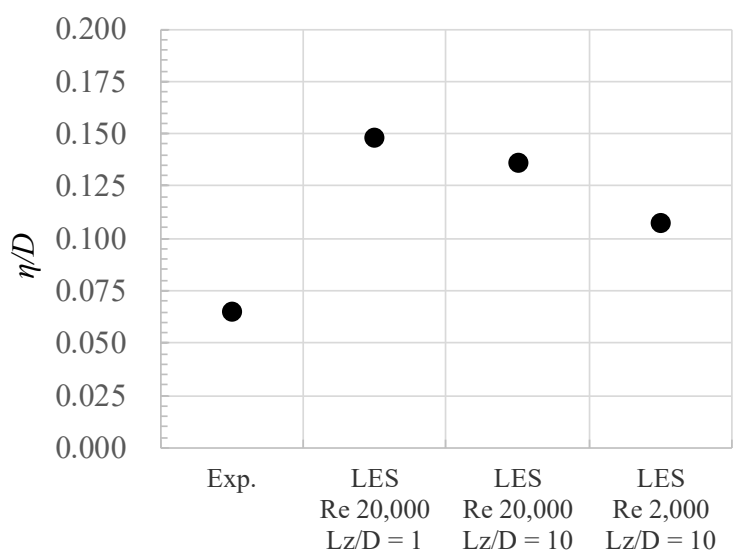

Fig. 17. VIV amplitude obtained by wind tunnel tests and numerical simulations using LES.

\section{Concluding remarks}

In this study, the amplitude of vortex-induced vibration (VIV) was calculated using the forced oscillation method with a large eddy simulation (LES) to avoid the large computational cost involved in the free vibration method, and to realize the practical use of a numerical simulation for the wind-resistant design of a long-span bridge. The conclusions are as follows:

- Flutter derivatives for aerodynamic damping obtained from the forced oscillation method using LES showed a clear dependence on the oscillation amplitude. Thus, the proposed method can calculate the VIV amplitude with a smaller computational cost than the free vibration method.

- A sufficiently large spanwise domain is required to consider the 3-D flow field for the proper evaluation of the aerodynamic damping and, consequently, the VIV amplitude.

- The Reynolds number changes the flow field around the bridge section to affect the aerodynamic damping and, thus, the VIV amplitude. The effect of Reynolds number is important, especially for reproducing the VIV amplitude obtained from wind tunnel tests with a small value of this number.

- The considered case in this study implies as a possibility that the VIV amplitude calculated by wind tunnel tests may be underestimated compared with that obtained from the prototype, because of a low Reynolds number resulting from the limitation of experimental facilities. In future work, investigations 
are necessary to explore the effects of the shape of the bridge section, including attachments such as handrails, on the dependence of the VIV amplitude on the Reynolds number.

- Even if the effects of the Reynolds number and the size of the spanwise domain were considered, the VIV amplitude obtained by the forced oscillation method using LES was still larger than that of the free vibration wind tunnel tests. The cause of this discrepancy should be investigated further to improve accuracy, for example, from the viewpoint of the effects of spanwise discretization and turbulent flow.

\section{Acknowledgment}

The supercomputer of ACCMS, Kyoto University, was used in this study for the flow simulation.

\section{References}

Álvarez, A.J., Nieto, F., Nguyen, D.T., Owen, J.S., Hernández, S. 2019. 3D LES simulations of a static and vertically free-to-oscillate 4:1 rectangular cylinder: Effects of the grid resolution. J. Wind Eng. Ind. Aerodyn. 192, 31-44. https://doi.org/10.1016/j.jweia.2019.06.012.

Bruno, L., Coste, N., Fransos, D. 2012. Simulated flow around a rectangular 5:1 cylinder: Spanwise discretisation effects and emerging flow features. J. Wind Eng. Ind. Aerodyn. 104-106, 203-215. https://doi.org/10.1016/j.jweia.2012.03.018.

Ferziger, J.H., Perić, M., 2002. Computational methods for fluid dynamics, 3rd edition. Springer, U.S.A. Guan, Q., Li, J., Guo, C., Liu, J., Xing, X., 2012. Effects of balusters on vortex-induced vibration of a bluff bridge deck section, Proc. 7th Int. Colloq. Bluff Body Aerodyn. Appl. 1078-1085.

Honshu-Shikoku Bridge Authority, 2001. Wind resistant design standard for Honshu-Shikoku bridges. Honshu-Shikoku Bridge Authority, Japan.

Ishihara, T., Oka, S., 2018. A numerical study of the aerodynamic characteristics of ice-accreted transmission lines. J. Wind Eng. Ind. Aerodyn. 177, 60-68. https://doi.org/10.1016/j.jweia.2018.04.008.

Ito, Y., Graham, J.M.R., 2016. LES for investigation of span-wise domain size effect and evaluation of flutter derivatives of a box bridge. Proc. 8th Int. Colloq. Bluff Body Aerodyn. Appl. 184.

Ito, Y., Graham, J.M.R., 2017. Large eddy simulation for static and unsteady wind forces of a box girder and the effect of span-wise domain size. J. Jpn. Soc. Civ. Eng. Ser. A1. 73 (1), 195-205. https://doi.org/10.2208/jscejseee.73.218.

Kajishima, T., 2014. Numerical simulation of turbulent flows, revised edition. Yokendo, Tokyo.

Laneville, A., Gartshore, I.S., Parkinson, G.V., 1975. An explanation of some effects of turbulence. Proc. 4th Int. Conf. on Wind Effects. Build. Struct. 333-341.

Maruoka, A., Hirano, H., 2000. Calculations of unsteady aerodynamic forces using CFD. J. Appl. Mech. 3, 707-712. https://doi.org/10.2208/journalam.3.707.

Matsumoto, M., Kobayashi, Y., Shirato, H., 1996. The influence of aerodynamic derivatives on flutter. J. Wind. Eng. Ind. Aerodyn. 60, 227-239. https://doi.org/10.1016/0167-6105(96)00036-0. 
553

554

555

556

557

558

559

560

561

562

563

564

565

566

567

568

569

570

571

572

573

574

575

576

577

578

579

580

581

582

583

584

585

586

587

588

589

590

Morinishi, Y., 1995. Conservative properties of finite difference schemes for incompressible flow. Trans. Annual Research Briefs, Center for Turbulence Research, Stanford University. 121-132.

Morse, T. L., Williamson, C. H. K., 2009. Prediction of vortex-induced vibration response by employing controlled motion. J. Fluid Mech. 634, 5-39. https://doi.org/10.1017/S0022112009990516.

Nagao, F., Utsunomiya, H., Yoshioka, E., Ikeuchi, A., Kobayashi, H., 1997. Effects of handrails on separated shear flow and vortex-induced oscillation. J. Wind Eng. Ind. Aerodyn. 69-71, 819-827. https://doi.org/10.1016/S0167-6105(97)00208-0.

Nguyen, D.T., Hargreaves, D.M., Owen, J.S., 2018. Vortex-induced vibration of a 5:1 rectangular cylinder: A comparison of wind tunnel sectional model tests and computational simulations. J. Wind. Eng. Ind. Aerodyn. 175, 1-16. https://doi.org/10.1016/j.jweia.2018.01.029.

Nieto, F., Owen, J., Hargreaves, D., Hernández, S., 2015. Bridge deck flutter derivatives: Efficient numerical evaluation exploiting their interdependence. J. Wind. Eng. Ind. Aerodyn. 136, 138- 150. http://doi.org/10.1016/j.jweia.2014.11.006.

Noguchi, K., Shirato, H., Yagi, T., 2017. Numerical evaluation of sea salt amounts deposited on bridge girders. J. Bridge Eng. 22(7). https://doi.org/10.1061/(ASCE)BE.1943-5592.0001061.

Okaze, T., Niiya, H., Nishimura, K., 2018. Development of a large-eddy simulation coupled with Lagrangian snow transport model. J. Wind Eng. Ind. Aerodyn. 183, 35-43. https://doi.org/10.1016/j.jweia.2018.09.027.

Sarpkaya, T., 1978. Fluid forces on oscillating cylinders. J. Waterway Port Coast. Ocean Div. ASCE. 104, 275-290.

Šarkić, A., Fisch, R., Höffer, R., Bletzinger, K.-U. U., 2012. Bridge flutter derivatives based on computed, validated pressure fields. J. Wind. Eng. Ind. Aerodyn. 104-106, 141-151. https://doi.org/10.1016/j.jweia.2012.02.033.

Sarwar, M.W., Ishihara, T., Shimada, K., Yamasaki, Y., Ikeda, T., 2008. Prediction of aerodynamic characteristics of a box girder section using the LES turbulence model. J. Wind Eng. Ind. Aerodyn. 96, 1895-1911. https://doi.org/10.1016/j.jweia.2008.02.015.

Sarwar, M.W., Ishihara, T., 2010. Numerical study on suppression of vortex-induced vibrations of box girder bridge section by aerodynamic countermeasures. J. Wind Eng. Ind. Aerodyn. 98 (12), 701711. https://doi.org/10.1016/j.jweia.2010.06.001.

Scanlan, R.H., Tomko, J.J., 1971. Airfoil and bridge deck flutter derivatives. J. Eng. Mech. Div. Proc. Am. Soc. Civ. Eng. 97 (6), 1717-1737.

Shimada, K., Ishihara, T., 2012. Predictability of unsteady two-dimensional k-E model on the aerodynamic instabilities of some rectangular prisms. J. Fluids Struct. 28, 20-39. https://doi.org/10.1016/j.jfluidstructs.2011.08.013.

Shiraishi, N., Matsumoto, M., 1983. On classification of vortex-induced oscillation and its application for bridge structure. J. Wind Eng. Ind. Aerodyn. 14, 419-430. https://doi.org/10.1016/01676105(83)90043-0.

Staubli, T., 1983. Calculation of the vibration of an elastically mounted cylinder using experimental data 
from forced vibration. ASME J. Fluids Eng. 105, 225-229. https://doi.org/10.1115/1.3240968.

Sun, D., Owen, J.S., Wright, N.G., Liaw, K.F., 2008. Fluid-structure interaction of prismatic line-like structures, using LES and block-iterative coupling. J. Wind Eng. Ind. Aerodyn. 96 (6-7), 840-858. https://doi.org/10.1016/j.jweia.2007.06.012.

Tamura, T., Miyagi, T., Kitagishi, T., 1998. Numerical prediction of unsteady pressures on a square cylinder with various corner shapes. J. Wind Eng. Ind. Aerodyn. 74-76, 531-542. https://doi.org/10.1016/S0167-6105(98)00048-8.

Washizu, K., Ohya, A., Otsuki, Y., Fujii, K., 1978. Aeroelastic instability of rectangular cylinders in a heaving mode. J. Sound Vib., 59(2), 195-210. https://doi.org/10.1016/0022-460X(78)90500-X.

Miyata. T., Miyazaki, M., Yamada, H., 1983. Pressure distribution measurements for wind induced vibrations of box girder bridges. J. Wind Eng. Ind. Aerodyn. 14(1-3), 223-234. https://doi.org/10.1016/0167-6105(83)90025-9.

Wardlaw, R.L., Tanaka, H., Utsunomiya, H., 1983. Wind tunnel experiments on the effects of turbulence on the aerodynamic behaviour of bridge road decks. J. Wind Eng. Ind. Aerodyn. 14(1-3), 247-257. https://doi.org/10.1016/0167-6105(83)90027-2.

Zhou, L., He, X., Jian, H., 2018. Numerical simulations and experimental validations on aerodynamic effect of wind barriers on train-bridge system. Proc. 7th Int. Conf. Comput. Wind Eng. 80. 